\title{
A Simple ImageJ-Based Method to Measure Cardiac Rhythm in Zebrafish Embryos
}

\author{
Bonifasius Putera Sampurna ${ }^{1}$, Gilbert Audira ${ }^{1}$, Stevhen Juniardi ${ }^{1}$, Yu-Heng Lai ${ }^{2, *}$ and \\ Chung-Der Hsiao 1,3,4,* ii \\ 1 Department of Bioscience Technology, Chung Yuan Christian University, 200 Chung-Pei Rd., \\ Chung-Li 32023, Taiwan; boni_bt123@hotmail.com (B.P.S.); gilbertaudira@yahoo.com (G.A.); \\ stvn.jun@gmail.com (S.J.) \\ 2 Department of Chemistry, Chinese Culture University, Taipei 11114, Taiwan \\ 3 Center of Nanotechnology, Chung Yuan Christian University, Chung-Li 32023, Taiwan \\ 4 Center of Biomedical Technology, Chung Yuan Christian University, Chung-Li 32023, Taiwan \\ * Correspondence: lyh21@ulive.pccu.edu.tw (Y.-H.L.); cdhsiao@cycu.edu.tw (C.-D.H.); \\ Tel.: +886-2-2861-0511 (Y.-H.L.); +886-3-265-3545 (C.-D.H.)
}

Received: 18 March 2018; Accepted: 3 April 2018; Published: 10 April 2018

\begin{abstract}
Zebrafish has an advantage of optical clarity within the body during its early developmental stage. Therefore, organs, including the heart, can be observed and analyzed in a non-invasive manner. Previously, heart rate measurement was analyzed by a complicated system, programming language, fluorescence transgenic zebrafish, or expensive setup that was not feasible and practical. In this study, we established a simple dissecting microscope with Charge-coupled Device (CCD) to capture the signal of heartbeat, and used ImageJ software as an open source platform to analyze cardiac rhythm for the atrium and the ventricle. We subjected astemizole to zebrafish embryos, and applied our apparatus to precisely detect heart arrhythmia (Atrium-Ventricle block) induction. In other words, we provided a simple, economical, and reliable method to measure cardiac rhythm in zebrafish embryos, which is able to evaluate whether cardiac rhythm is affected by chemical compounds.
\end{abstract}

Keywords: ImageJ; region of interest (ROI); cardiac rhythm; arrhythmia; zebrafish

\section{Introduction}

Zebrafish is a tropical Cyprinid teleost fish that has been widely applied to developmental studies [1]. Taking advantage of body transparency, the developmental process of organs, such as the heart, brain, gills, muscles, blood etc., can be visually identified during the embryonic stage [2]. Zebrafish develops discrete organs and tissues, including the brain, liver, pancreas, kidneys, intestines, bones, muscles, nerve systems, and sensory organs, within 120 hour-post-fertilization (hpf). In addition, within $26 \mathrm{hpf}$, zebrafish shows a beating heart controlled by both a complex network of ion channels and functional metabolism. Moreover, the early embryonic development is much more difficult to examine and may need an invasive approach to identify it in mammals. Therefore, the quick developing time frame of zebrafish indeed facilitates in research observation and demonstration.

The heart, one of the most important organs in zebrafish, has two chambers: one atrium and one ventricle [3]. The pumping mechanism of contraction and relaxation within the heart is similar in both cellular and molecular levels between fish and mammals [4,5]. These similarities include: (1) blood flows from sinus venosus into an atrium; (2) blood moves through the ventricle to the aorta; (3) valves direct blood flow; (4) specialized endocardium musculature drives a high-pressure system; (5) an electrical system regulates heartbeat rhythm; and (6) a pacemaker is associated with 
heartbeat regulation. Therefore, studies on heart development and physiology with a fish model can help us to understand the mechanism in mammals. In addition, due to its small body size and large productivity, fish are an ideal model to perform high-throughput drug screening to identify potential chemicals that alter heartbeat rhythm [6,7]. Moreover, the transparency of the embryonic zebrafish facilitates rapid evaluation of heart rate and rhythm. Previously, several approaches were developed to monitor heartbeat. For example, transgenic cardiomyocyte and blood cell lines with fluorescent signals were applied to measure heartbeat rhythm [8]. Dual-beam optical reflectometry was used to measure cardiac rhythm in non-label zebrafish embryos [9]. Moreover, a power spectral method was used to determine heartbeat rhythm by calculating the dynamic pixel change from caudal blood vessel circulation in embryos [10]. Various techniques have been developed; however, the methods were still impeded by some limitations. For example, first, programming scripts were required for most methods to conduct the calculation $[8,10]$. Second, generation of transgenic lines was time-consuming in order to optimize signal-to-noise ratio [8]. Third, expensive apparatuses were required to capture heartbeat images or videos [9]. Therefore, it is urgent to develop an alternative and simple assay to evaluate zebrafish cardiac rhythm.

Arrhythmia is an irregular heart rhythm syndrome, which is either due to genetically inherited mutations of ion channels or, more commonly, affected by drugs that inhibited cardiac repolarization. The syndrome can be self-limited or developed into ventricular fibrillation, which leads to rapid death [6]. There are three categories of rhythm disturbance: irregular, fast (tachycardia), and slow (bradycardia) [11]. Astemizole, known as HERG (human ether-a-go-go-related gene) blocking compounds, was able to induce rapid, highly specific, and reproducible arrhythmia [12]. Zebrafish ERG (ZERG) was already expressed during the early developmental stages of zebrafish. A highly conserved amino acid sequence of the pore-forming domain was identified within zebrafish and human ERG [13]. In this study, we developed a simple and economical method to measure cardiac rhythm in wild-type fish embryos without fluorescent labeling. Our new technique was also validated by evaluating astemizole on blocking A-V transduction to induce arrhythmia.

\section{Materials and Methods}

\subsection{Zebrafish Maintenance and Sample Preparation}

Zebrafish embryos were raised and kept in the incubator at $28^{\circ} \mathrm{C}$ as described [14]. A wild type AB strain of zebrafish was used in this experiment. Embryos aged 72-h-post-fertilization (hpf) were used to perform heartbeat measurement before being covered by pigments. A pigment formation inhibitor of PTU (1-phenyl 2-thiourea) was not applied in our experiment in order to avoid any possible side effects on cardiac rhythm. MS-222 (tricaine mesylate) was not used because of its induction effect on heart rate [15] and synergistic effect with other compounds [16]. Healthy embryos were selected and moved to 6-cm plastic Petri dishes. Excessive water was removed before 3\% methylcellulose was used to mount the fish. The animal experiments followed the protocol and was approved by the Institutional Animal Care and Use Committee of Chung Yuan Christian University with approval number 106025 (25 May 2017).

\subsection{Video Recording}

Our analysis including video capture, image transformation, and dynamic pixel changes to heartbeat rhythm calculation were presented in Figure 1A. The temperature in chamber was maintained at $28{ }^{\circ} \mathrm{C}$. The position of the fish was adjusted to the anterior-to-left position, which can be manipulated by using an XY stage (Figure 1B). Video was captured by a digital charge-coupled device (CCD) (SK2700HDMI-T2, Zgenebio, Taipei, Taiwan) mounted on a dissecting microscope at a speed of 60 frames per second (fps), with $1920 \times 1080$ pixels resolution. The CCD was connected to a television with an High Definition Multimedia Interface (HDMI) cable to monitor fish position and heartbeat, and this was controlled remotely. Heart chronological analysis was examined by directly recording the 
video after the fish position was fixed and a compound was added. A thirty-minute recording period with the same video setting as above was used using a chronological method.
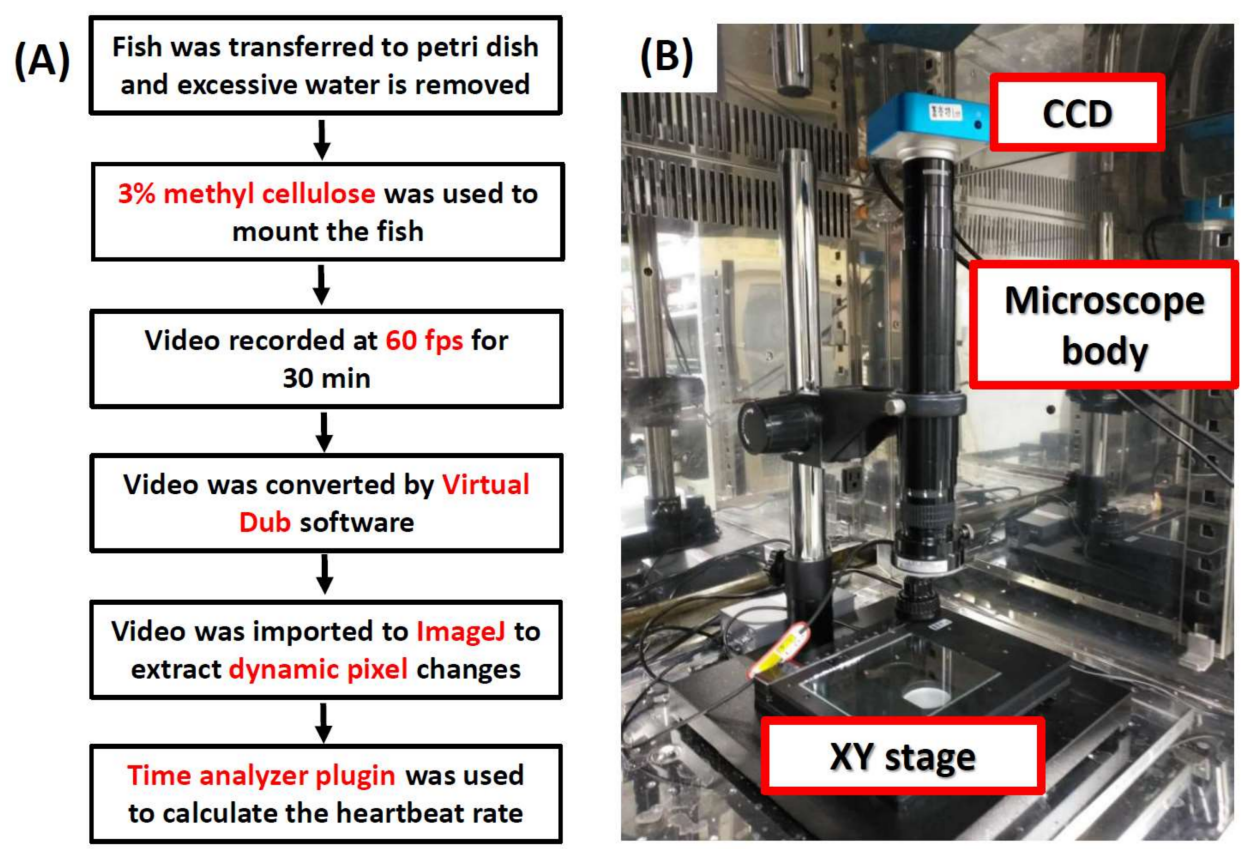

Figure 1. The equipment setting and analysis pipeline to detect cardiac rhythm in zebrafish embryos. (A) flowchart of analyzing cardiac rhythm in zebrafish embryos by using the dynamic pixel change method. Software Adobe Premiere, VirtualDub, ImageJ, Microsoft Excel, GraphPad Prism6 and Origin 9.1 were applied in our assay; (B) to avoid temperature fluctuation during the experiment, one dissecting microscope mounted with a Charge-coupled Device (CCD) was installed in a temperature controlled incubator.

\subsection{Video Processing}

Time point of interest $(0,5,10,15,20,25$ and $30 \mathrm{~min}$ after drug exposure) was selected for recording at $10 \mathrm{~s}$ duration and exported by using Adobe Premiere CC software (Available online: http://www.adobe.com/products/premiere.html). The video recording frame rate was reduced to $30 \mathrm{fps}$ and a multimedia container format changed to AVI format after processed with Adobe Premiere CC. Video was rendered with VirtualDub software (Available online: http:/ / www.virtualdub.org / ) to make it compatible with ImageJ software (Available online: https://imagej.nih.gov/ij/). X264vfw software (Available online: https://sourceforge.net/proj ects/x264vfw/files/x264vfw64/) needs to be installed to have VirtualDub recognize the Audio Video Interleave (AVI) video file. ImageJ was used as the major platform for heart rate analysis in this study. Region of interest (ROI) was selected by using a circle tool. Selection of ROI can be specified within the atrium, the ventricle or the whole heart regions. Not only red blood cells, but also external factors such as pigments and debris would affect average pixel intensity with this approach. Therefore, it is important not to select ROI in the heart region that may make noise (Figure S1). In addition, fish embryos floating in the mounting medium may lead to pixel intensity pattern shift (Figure S1). However, this pixel change pattern shift still could be analyzed to extract the heartbeat frequency. A time series analyzer V3 plugin (Available online: https: / /imagej.nih.gov/ij/plugins/time-series.html) was used directly to analyze the dynamic pixel changes in the selected ROI. The "Add button" was clicked to choose the ROI that has been selected. "Get average" was clicked to get the peak and table data. The table data were exported and saved in Excel format for downstream analysis. The detail operating process can be found in the supplementary protocol. 


\subsection{Time Interval and BPM Analysis}

Frame was converted into time by dividing the frame rate. Graphic smoothing of heartbeat rhythm was done by Origin 9.1 software (Originlab Corporation, Northampton, MA, USA) with the default setting. Peak Analyzer function in Origin 9.1 was used to calculate time of the peak. Peak of Center (X\&Y) in Plot_Data was processed by Microsoft Excel (Microsoft 2016 version, Seattle, WA, USA) to calculate time interval and beat per minute (BPM). Heartbeat time interval was calculated by subtracting two consecutive time points. BPM was obtained by dividing one minute (60 s) by the time interval. Heartbeat frequency was done by short-time Fourier transform (STFT) in Signal Processing in Origin 9.1 software with default settings.

\subsection{Drug Treatment}

Astemizole (Sigma, A2861, St. Louis, MO, USA) was used as an arrhythmia-inducing drug to examine zebrafish heartbeat. In addition, $10 \mathrm{mM}$ stock solution of astemizole was prepared with $100 \%$ Dimethyl sulfoxide (DMSO) and diluted with distilled water to make a working solution at $5 \mu \mathrm{M}$. In chronic treatment, $10 \mu \mathrm{M}$ astemizole was dropped onto the fish mounted with the same volume (around $50 \mu \mathrm{L}$ ) of $3 \%$ methylcellulose to the final concentration of $5 \mu \mathrm{M}$.

\subsection{Statistical Analysis}

Statistical and graphic analysis were operated by GraphPad Prism 6 software (Available online: https:/ / www.graphpad.com/scientific-software/prism/). A column table option was used to enter the data and enter replicate values, stacked into columns. Data in endpoint and chronological methods were presented as mean \pm SEM, and a $t$-test (with parametric assay) was used to calculate the significance. An unpaired student $t$-test was applied to compare the significance within two groups. Sample distribution was assumed to be normally distributed. A difference between two means was significant when ${ }^{*} p<0.05,{ }^{* *} p<0.005,{ }^{* * *} p<0.001$ and ${ }^{* * * *} p<0.0001$.

\section{Results}

\subsection{Cardiac Rhythm Can Be Detected by the Dynamic Pixel Method}

Our cardiac rhythm measurement method was based on the dynamic pixel changes in the heart region (Figure 2A). Most images had a valid intensity range of pixels from 0 (black) to 255 (white). The higher the brightness of an image, the higher the pixel intensity number [17]. The highest peak represented the greatest abundance of red blood cells that were pumped out of the heart chamber, defined as contraction (systolic phase). On the other hand, the lowest peak represented the greatest abundance of red blood cells that filled the heart chamber, defined as relaxation (diastolic phase) (Figure 2B,C). Generally, a normal human heart rate is around 60-90 beats-per-minute (bpm) in the resting state. The heart rate of $72 \mathrm{hpf}$ zebrafish is around $120-180 \mathrm{bpm}$. Therefore, zebrafish is an ideal animal model for heart rate compared to mice with high heart rates at 300-600 bpm [8]. We observed that the heart rate of wild-type fish was at $137 \pm 8 \mathrm{bpm}(n=10)$. Based on either the atrium or the ventricle as ROI calculation, we tracked the cardiac rhythm and found there were no significant differences between the atrium and the ventricle (Figure 2C). The distance between two consecutive highest points of the peaks was calculated as time interval of contraction. The time interval of atrium and ventricle contraction was consistently at $0.4 \pm 0.03 \mathrm{~s}$ in control embryos at $72 \mathrm{hpf}$. Short-Time Fourier Transformation (STFT) was used to transform time interval into frequency domain (Figure 2D). The heartbeat frequency in control fish was around $2.3 \mathrm{~Hz}$ (Figure 2D), which is consistent with previous study $[18,19]$. Basic frequency (red color) in wild-type control was consistent during the examination period [10]. 

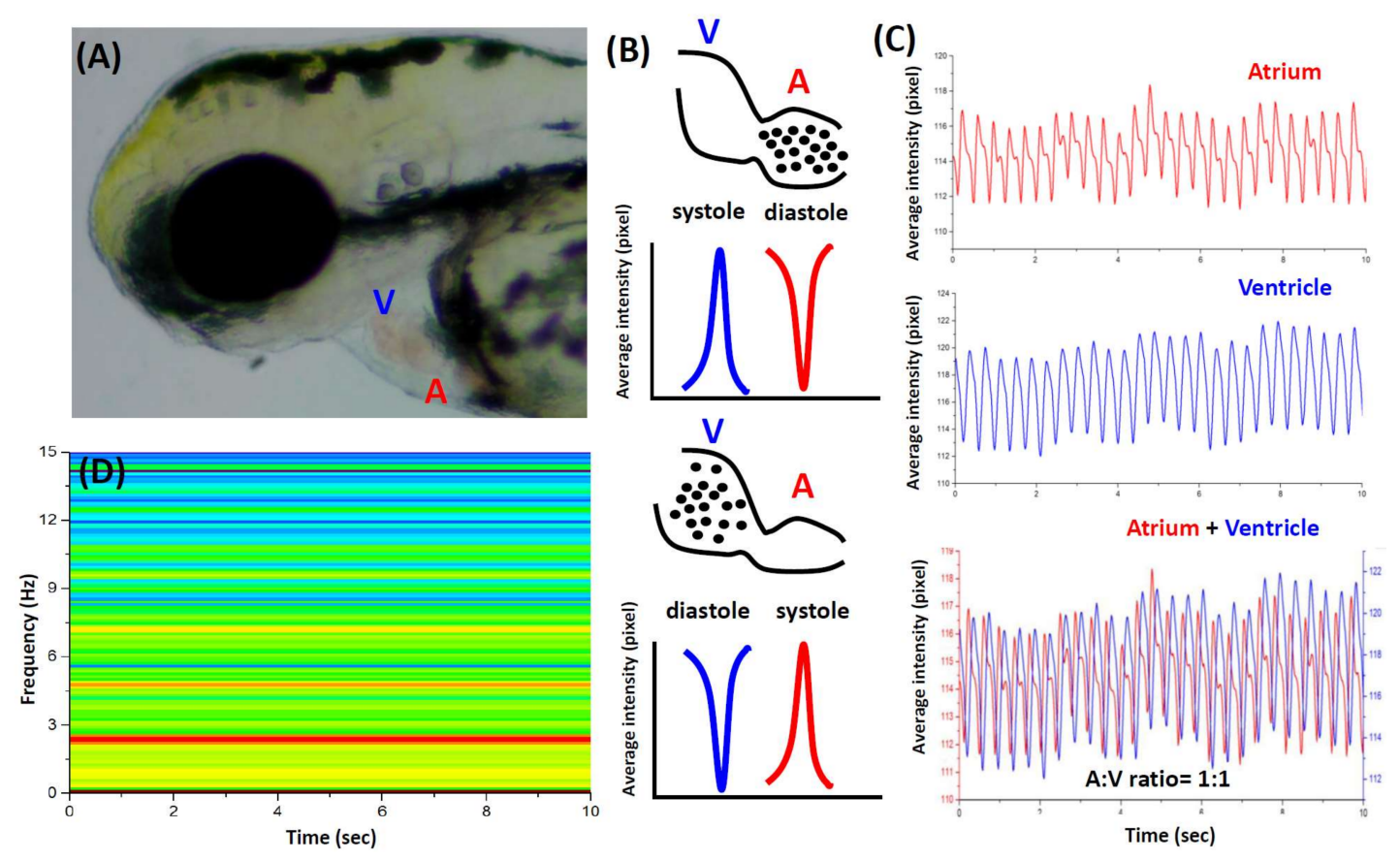

Figure 2. Cardiac rhythm detected by dynamic pixel change method. (A) the heart position of zebrafish embryos aged $72 \mathrm{hpf}$ (B) schematic diagram showing the principle of the dynamic pixel-changing method used in this study; (C) the dynamic pixel change pattern in the atrium (upper panel), the ventricle (middle panel) and both (lower panel) for the zebrafish aged $72 \mathrm{hpf}$; (D) short time Fourier transformation of heartbeat for untreated zebrafish embryos aged $72 \mathrm{hpf}(\mathrm{V}$, ventricle; A, atrium).

\subsection{Endpoint Method of Astemizole Incubation}

Next, we evaluated the potential effect of astemizole on heartbeat regularity in zebrafish embryos aged $72 \mathrm{hpf}$ to validate our design. Astemizole is the second generation of antihistamines, which was used to treat the majority of allergic diseases, especially allergic conjunctivitis, urticaria, and anaphylaxis [20]. However, it has already been withdrawn from the market due to the cardiotoxicity effect [21]. During electrocardiogram (ECG) detection in human patients, astemizole displayed long QT intervals, arrhythmia that triggered torsades de pointes, and caused sudden death [22]. We observed that embryonic zebrafish changed the cardiac rhythm within 1 hour after being treated with $5 \mu \mathrm{M}$ astemizole by an endpoint evaluation method (see Video S1 for wild-type and Video S2 for astemizole-treated embryos). Atrium contraction rate was significantly decreased to $77 \pm 17 \mathrm{bpm}$ $(n=10)$, compared with the control atrium contraction rate at $137 \pm 8 \mathrm{bpm}$ (Figure 3A). The time interval of two consecutive atrium beats in $5 \mu \mathrm{M}$ astemizole-treated group was increased to $0.82 \pm 0.2 \mathrm{~s}$ compared to control group at $0.44 \pm 0.03 \mathrm{~s}$ (Figure 3C). This indicated that astemizole treatment prolonged the contraction interval, which was consistent with ECG results of prolongation on QT interval. Moreover, the ventricle stopped contracting after $5 \mu \mathrm{M}$ astemizole treatment for $1 \mathrm{~h}$ (Figure 3B,D). It suggested that rapid blockage of astemizole was due to activation of delayed rectifier $\mathrm{K}^{+}$current $\left(\mathrm{I}_{\mathrm{Kr}}\right)$ or homologue of the human ether-a-go-go-related gene (HERG) $\mathrm{K}^{+}$channels, which is important for the repolarization phase during cardiac regulation [23]. On the other hand, explanation from the structural viewpoint for the astemizole blocking $\mathrm{K}^{+}$channel was because of its interaction with two special aromatic amino acids on the S6 domain of the channel [12]. 


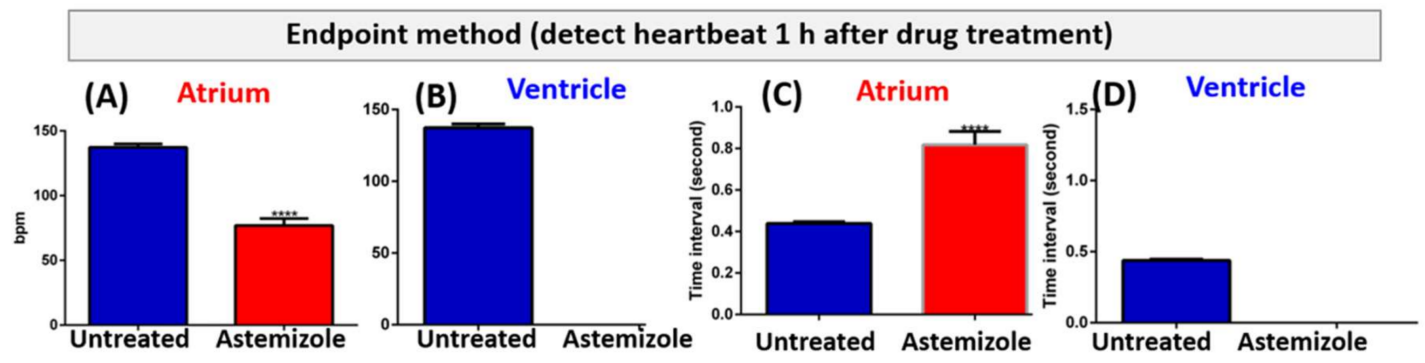

Time Chronology method (detect heartbeat continuous after drug treatment)

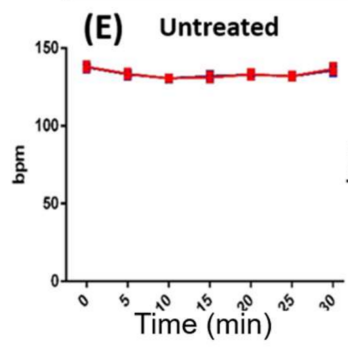

(I) Astemizole Omin

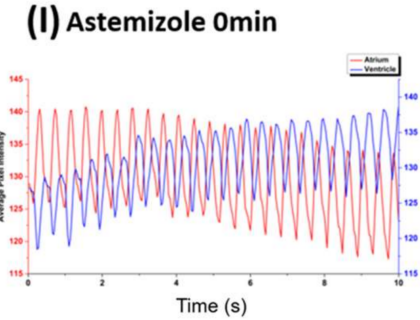

(F) Astemizole

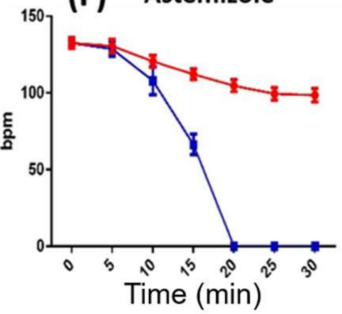

(J) Astemizole 10min

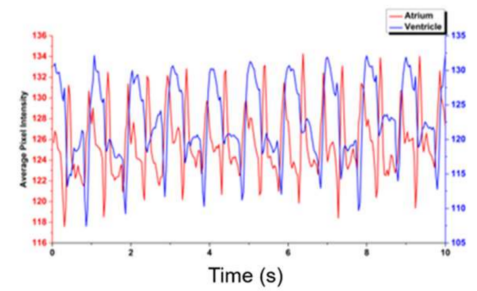

(G) Untreated
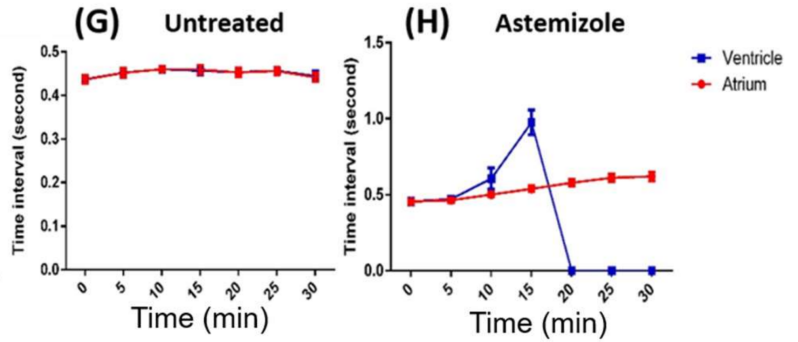

(L)Astemizole 20min

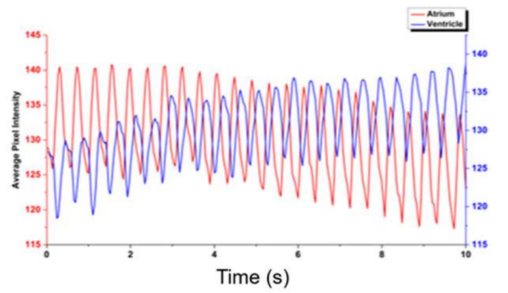

Figure 3. Endpoint and time chronological evaluation of astemizole on heartbeat in embryonic zebrafish 72 hpf. Atrium (A,B) and ventricle beat-per-minute (bpm) were compared between untreated control and $5 \mu \mathrm{M}$ astemizole-treated embryos after one hour of incubation. Atrium (C) and ventricle (D) beating time interval of untreated control and $5 \mu \mathrm{M}$ astemizole-treated embryos after one hour of incubation. Beat-per-minute (E) and time interval (G) in untreated control embryos in a chronological method. Beat-per-minute (F) and time interval $(\mathbf{H})$ in $5 \mu \mathrm{M}$ astemizole-treated embyros in a chronological method; (I-K) is the dynamic pixel change patterns of the atrium and the ventricle after 0,10 and $20 \mathrm{~min}$ astemizole treatment at $5 \mu \mathrm{M}$ concentration. The data are expressed as the means \pm SEM and were analyzed by $t$-test $(* * * * 0.0001, n=10)$.

\subsection{Time Chronology Method of Astemizole}

During the previous endpoint approach, we found that the ventricle contraction had already stopped after $5 \mu \mathrm{M}$ astemizole incubation for 1 hour. Therefore, we attempted to use chronological techniques to detect the atrioventricular blockage for a long time period. To ensure our chronological method was feasible, 10 wild-type embryos aged at $72 \mathrm{hpf}$ were used as control. In the control chronological analysis, there was no significant difference between the atrium and the ventricle for each time point (Figure 3E,G). This result indicated that the time chronology method was solid and precise in order to analyze cardiac rhythm change in zebrafish embryos. In contrast, after subjection to $5 \mu \mathrm{M}$ astemizole, the heart still demonstrated normal beating within the first $5 \mathrm{~min}$. At 0-time point, the atrium and the ventricle had average contraction at $133 \pm 12 \mathrm{bpm}$. After $5 \mathrm{~min}$, the atrium had contraction at $131 \pm 14 \mathrm{bpm}$ and the ventricle had contraction at $129 \pm 16 \mathrm{bpm}$. The atrium and ventricle showed more arrhythmia from 10 min onwards, for which the atrium had $121 \pm 12 \mathrm{bpm}$ and the ventricle had $108 \pm 29 \mathrm{bpm}$ rhythm. The arrhythmia was more severe 15 min post treatment with the atrium at $112 \pm 11 \mathrm{bpm}$ and the ventricle at $66 \pm 22 \mathrm{bpm}$. Twenty minutes later, the atrium had $104 \pm 12 \mathrm{bpm}$ rhythm; however, the ventricle was no longer pumping, only quivering instead (Figure 3F). In $20 \mathrm{~min}$, atrium contraction was reduced $22 \%$ from 
the beginning (Figure 3F). After 15 min, ventricular contraction was completely blocked (Figure 3F,H). Losing ventricular contraction causes blood circulation to be interrupted and blood cells accumulated in the pericardiac chambers. The phenomenon of lacking ventricle contraction and blood propagating backwards into the atrium were consistent with previous study [24]. Although the heart lacked blood circulation in 20 min (Figure 3F,H), zebrafish embryos still survive for over 30 min. Dynamic pixel change patterns showed chronological changes from the 0 to $20 \mathrm{~min}$ time period after astemizole administration (Figure 3I-K).

\section{Discussion}

Several methods have already been established to analyze heart rate in zebrafish $[8-10,25,26]$. Compared to previous methods, our method provides several advantages (summarized in Table 1). First, specific transgenic fish are not required to detect heart rate, since expression or deletion of genes may increase or decrease sensitivity to drugs causing QT prolongation [12]. Second, expensive set up of a high-speed confocal microscope is not required to capture the heart movement in fluorescent transgenic fish. In our method, only a conventional dissecting microscope and CCD are required to record video. Third, treating with anaesthesia and pigment blocking could cause an interaction effect with tested chemical compounds [16]. In our protocol, only 3\% methylcellulose was used to mount embryos and eliminate the potential bias from drug cross-reaction. Fourth, in our protocol, atrium, ventricle, and whole heart contraction information were collected from a single video recording in one demonstration. Compared to previous studies, zebrafish heartbeat calculations were mainly focused on the whole heart beating, while the atrium and ventricle beating rhythm was not addressed very much $[10,25]$. In our method, the potential drugs for atrioventricular blockage could be studied in detail by this image-based, time chronology method. According to our findings, the atrium and ventricular beating ratio is 1:1 in wild-type zebrafish embryos, while astemizole leads to a 2:1 atrioventricular block at initial and, finally, total block ventricle beating, which showed a significant difference between these two groups. Our current protocol is valid at embryonic or larvae stages. Zebrafish heart chambers are developed and differentiated at $48 \mathrm{hpf}$ and the atrium and ventricle are able to be identified in this developmental stage. Unfortunately, the cardiac rhythm in adult fish cannot be detected by using our method since the pericardial cavity is non-transparent [27].

ImageJ is an easy operation open source platform to analyze biological data for scientists without coding training background. In addition, all plugin functions for ImageJ that are used in our method can be downloaded for free. This platform is adaptable with no requirement of script writing to execute the program. Moreover, compared to some manual written scripts in MATLAB that need to be updated periodically, the Time Series Analyzer plugin in this method created in 2006 (Available online: https:/ /imagej.nih.gov/ij/plugins/time-series.html) can still be used in the latest ImageJ version. Otherwise, it becomes incompatible with the newer version of MATLAB [26]. One disadvantage of the currently provided cardiac rhythm measurement method is using commercial software such as Adobe Premiere, Origin 9.1, and GraphPad Prism 6, and this might become costly. Free video editing software could be used as alternative from Adobe Premiere. For example, time interval and beat per minute analysis by Origin 9.1 could be replaced by Microsoft Excel software. Graphic and statistical analysis function also could be done by Microsoft Excel. The reason that we used commercial software because of its features provided rapid data collection and more professional graphic and statistical presentation.

Generally, repolarization of the cardiac myocyte was driven by normal function of channels, receptors, and cytoskeletal proteins [28]. Previous studies showed that astemizole prolonged QT on the human heart rate, and consistently exhibited bradycardia in zebrafish heart [29]. Many antiarrhythmics and non-cardiac drugs have been reported to cause QT prolongation and/or Torsades de pointes, which indicated that the great concern of the risk of pro-arrhythmia drugs during clinical therapeutic application [30]. Heart arrhythmia induced by astemizole was confirmed and has been withdrawn by the FDA in 1999 [21]. Astemizole is a second-generation histamine (H1)-receptor antagonist to treat an allergic reaction. One clinical case showed that, seven hours after subjecting astemizole (total dose 
$200 \mathrm{mg}$ ), the child became unconscious and found no palpable cardiac output. Although the child was recovered by an intensive therapy unit with a normal heart rate and blood pressure, ventricular tachyarrhythmia developed spontaneously one hour later [24]. Furthermore, when a bioengineered human cardiac organoid examined the drugs that caused a prolonged QT syndrome, astemizole showed the most severe heartbeat alteration among the drugs tested [31].

A long QT syndrome can be caused by multiple mutations on ion currents including $\mathrm{I}_{\mathrm{Ks}}, \mathrm{I}_{\mathrm{Kr}}$, and $\mathrm{I}_{\mathrm{Na}}$. On the other hand, drugs that prolong QT interval block the potassium channel [32]. HERG and its homologue gene encode a pore-forming $\alpha$ subunit for $\mathrm{I}_{\mathrm{Kr}}$. HERG-associated channel inhibition has been identified relative to QT prolongation. Astemizole acts as a potent HERG channel blocker, which induced arrhythmia by detaining rectifier $\mathrm{I}_{\mathrm{Kr}} \mathrm{K}^{+}$channel (KCNH2) [33]. KCNH2 is critical to repolarization of the heart chamber. Astemizole caused a reduction of atrium contraction around $22 \%$ from the normal atrium contraction after being treated for $1 \mathrm{~h}$. The ventricle did not contract anymore after long incubation. The spontaneous tachycardia effect of astemizole can also be studied in detail with the zebrafish model by using our method. Astemizole is rapidly metabolized in the body, which inferred that the ventricle is always affected more than the atrium. Therefore, we hypothesized that the ventricle of the zebrafish embryo depends more on $\mathrm{I}_{\mathrm{Kr}}$ repolarization than the atrium [12]. The chronological method can be done in $30 \mathrm{~min}$ to show the dynamics of alteration in heart contraction. Within $10 \mathrm{~min}$ after incubation, we found that the atrium and the ventricle already generated different contraction/relaxation time intervals. After $15 \mathrm{~min}$, the difference in the time interval between the atrium and the ventricle became more significant. Finally, by the 20-min mark, the ventricle had already stopped beating and was only quivering. Not only spontaneous ventricular tachyarrhythmia, but also red blood cells flow, chamber contraction, and heart rate changes were able to be demonstrated in this approach.

Sudden cardiac death (SDC) caused around 184,000-462,000 cases per year in the United States. One of the onsets of SDC is the response of exogenous factors, such as drugs and metabolite disturbance. Multi-purpose drugs, such as antimicrobial, antihistamine, and psychoactive agents, could induce arrhythmia that leads to SDC. Withdrawal of astemizole (1999), grepafloxacin (1999), cisapride (2000), thioridazine (2005), and propoxyphene (2010) from the market were due to stimulation of the QT prolonged syndrome [34]. Many of the approved drugs have not been screened for QT prolonged potential [35]. Therefore, new compounds must undergo a cardiotoxicity assay to reduce the arrhythmia effect and developing and finding a well-established approach is essential to the pharmaceutical market.

\section{Conclusions}

In conclusion, our method provides an easy and low-cost screening for cardiotoxicity drugs in zebrafish embryos with a novel signaling interpretation. By using the video analysis method in Image instead of running scripts language, it can greatly reduce the operating difficulty for most biologists who do not have much of a background in programming. In addition, the data obtained from the ImageJ platform were highly qualified and competitive compared with other approaches. It may apply to further screening for new compounds that cause heart rate alteration. Manual calculation of time interval and heartbeat per minute was done in Origin 9.1 combined with Excel software. Currently, user intervention must be developed into an automatic process in every field. Therefore, developing macro language and increasing the number of dissecting microscopes and CCD that are operated automatically in parallel is a definite trend and will be able to improve the throughput of this assay. 
Table 1. Comparison of different methods to measure heartbeat regularity in zebrafish.

\begin{tabular}{|c|c|c|c|c|c|c|}
\hline Parameter & This Study & De Luca et al. [8] & Lai et al. [9] & Yozzo et al. [25] & Fink et al. [26] & Chan et al. [10] \\
\hline $\begin{array}{l}\text { Require special } \\
\text { transgenic line? }\end{array}$ & No & Yes & No & Yes & No & No \\
\hline $\begin{array}{l}\text { Require special script to } \\
\text { run software? }\end{array}$ & No & Yes & Yes & Yes & Yes & Yes \\
\hline $\begin{array}{l}\text { Major platform to } \\
\text { calculate heartbeat } \\
\text { regularity }\end{array}$ & ImageJ & MATLAB & $\begin{array}{l}\text { Not reported in original } \\
\text { article }\end{array}$ & $\begin{array}{l}\text { MetaXpress 4.0.0.24 } \\
\text { software (commercial) }\end{array}$ & MATLAB & $\begin{array}{l}\text { A custom-made } \\
\text { program which } \\
\text { developed in C\# (C } \\
\text { sharp) language was } \\
\text { used for digital motion } \\
\text { analysis }\end{array}$ \\
\hline $\begin{array}{l}\text { Require expensive } \\
\text { setup to capture } \\
\text { heartbeat video? }\end{array}$ & No & Yes & Yes & Yes & Yes & No \\
\hline $\begin{array}{l}\text { Major facility to capture } \\
\text { heartbeat images }\end{array}$ & $\begin{array}{l}\text { CCD mount onto } \\
\text { dissecting microscope }\end{array}$ & $\begin{array}{l}\text { Leica TCS SP5X II } \\
\text { confocal laser-scanning } \\
\text { inverted microscope } \\
\text { equipped with a } \\
\text { tandem scanning } \\
\text { system }\end{array}$ & $\begin{array}{l}\text { Dual-beam optical } \\
\text { reflectometry set up in } \\
\text { inverted microscope }\end{array}$ & $\begin{array}{l}\text { ImageXpress Micro } \\
\text { (IXM) Widefield } \\
\text { High-Content } \\
\text { Screening }\end{array}$ & $\begin{array}{l}\text { Hamamatsu EM-CCD } \\
\text { digital camera mounted } \\
\text { on Leica DM-LFSA } \\
\text { microscope }\end{array}$ & $\begin{array}{l}\text { CCD mount onto } \\
\text { dissecting microscope }\end{array}$ \\
\hline $\begin{array}{l}\text { Position to capture } \\
\text { heartbeat information }\end{array}$ & Heart & Heart & Heart & Heart & Heart & Caudal blood vessel \\
\hline $\begin{array}{l}\text { What kind of message } \\
\text { can be obtained? }\end{array}$ & $\begin{array}{l}\text { Atrium and ventricle } \\
\text { rhythm, whole } \\
\text { heartbeat frequency }\end{array}$ & $\begin{array}{l}\text { Atrium and ventricle } \\
\text { rhythm, whole } \\
\text { heartbeat frequency }\end{array}$ & $\begin{array}{l}\text { Atrium and ventricle } \\
\text { rhythm, whole } \\
\text { heartbeat frequency }\end{array}$ & $\begin{array}{l}\text { Whole heartbeat } \\
\text { frequency }\end{array}$ & $\begin{array}{l}\text { Atrium and ventricle } \\
\text { rhythm, whole } \\
\text { heartbeat frequency }\end{array}$ & $\begin{array}{l}\text { Whole heartbeat } \\
\text { frequency }\end{array}$ \\
\hline
\end{tabular}


Supplementary Materials: The following are available online at http://www.mdpi.com/2411-5134/3/2/21/s1, Figure S1: The schematic picture showing the movement of fish during video recording will cause dynamic pixel alteration, but still can extract the heartbeat frequency from the recorded videos, Video S1: The heartbeat in wild-type zebrafish embryos aged at $72 \mathrm{hpf}$, Video S2: The heartbeat in astemizole-treated zebrafish embryos aged at $72 \mathrm{hpf}$.

Acknowledgments: This work was funded by Ministry of Science and Technology (grant no. MOST105-2313-B-033-001-MY3) to Chung-Der Hsiao.

Author Contributions: Yu-Heng Lai and Chung-Der Hsiao conceived and designed the experiments; Bonifasius Putera Sampurna and Gilbert Audira performed the experiments; Stevhen Juniardi analyzed the data; Bonifasius Putera Sampurna, Yu-Heng Lai and Chung-Der Hsiao wrote the paper.

Conflicts of Interest: The authors declare no conflict of interest.

\section{References}

1. Barrionuevo, W.; Burggren, $\mathrm{W} . \mathrm{O}_{2}$ consumption and heart rate in developing zebrafish (Danio rerio): Influence of temperature and ambient $\mathrm{O}_{2}$. Am. J. Physiol. Regul. Integr. Comp. Physiol. 1999, 276, R505-R513. [CrossRef]

2. Fishman, M.C.; Stainier, D.Y.; Breitbart, R.E.; Westerfield, M. Zebrafish: Genetic and embryological methods in a transparent vertebrate embryo. Methods Cell Boil. 1997, 52, 67-82.

3. Stainier, D.Y. Zebrafish genetics and vertebrate heart formation. Nat. Rev. Genet. 2001, 2, 39-48. [CrossRef] [PubMed]

4. Nemtsas, P.; Wettwer, E.; Christ, T.; Weidinger, G.; Ravens, U. Adult zebrafish heart as a model for human heart? An electrophysiological study. J. Mol. Cell. Cardiol. 2010, 48, 161-171. [CrossRef] [PubMed]

5. Staudt, D.; Stainier, D. Uncovering the molecular and cellular mechanisms of heart development using the zebrafish. Ann. Rev. Genet. 2012, 46, 397-418. [CrossRef] [PubMed]

6. Milan, D.J.; Peterson, T.A.; Ruskin, J.N.; Peterson, R.T.; MacRae, C.A. Drugs that induce repolarization abnormalities cause bradycardia in zebrafish. Circulation 2003, 107, 1355-1358. [CrossRef] [PubMed]

7. Burns, C.G. High-throughput assay for small molecules that modulate zebrafish embryonic heart rate. Nat. Chem. Boil. 2005, 1, 263-264. [CrossRef] [PubMed]

8. De Luca, E. ZebraBeat: A flexible platform for the analysis of the cardiac rate in zebrafish embryos. Sci. Rep. 2014, 4, 4898. [CrossRef]

9. Lai, Y.-C.; Chang, W.-T.; Lin, K.-Y.; Liau, I. Optical assessment of the cardiac rhythm of contracting cardiomyocytes in vitro and a pulsating heart in vivo for pharmacological screening. Biomed. Opt. Express 2014, 5, 1616-1625. [CrossRef] [PubMed]

10. Chan, P.K.; Lin, C.C.; Cheng, S.H. Noninvasive technique for measurement of heartbeat regularity in zebrafish (Danio rerio) embryos. BMC Biotechnol. 2009, 9, 11. [CrossRef] [PubMed]

11. Skinner, J.R.; Sharland, G. Detection and management of life threatening arrhythmias in the perinatal period. Early Hum. Dev. 2008, 84, 161-172. [CrossRef] [PubMed]

12. Langheinrich, U.; Vacun, G.; Wagner, T. Zebrafish embryos express an orthologue of HERG and are sensitive toward a range of QT-prolonging drugs inducing severe arrhythmia. Toxicol. Appl. Pharmacol. 2003, 193, 370-382. [CrossRef] [PubMed]

13. McGrath, P.; Li, C.-Q. Zebrafish: A predictive model for assessing drug-induced toxicity. Drug Discov. Today 2008, 13, 394-401. [CrossRef] [PubMed]

14. Avdesh, A. Regular care and maintenance of a zebrafish (Danio rerio) laboratory: An introduction. J. Vis. Exp. 2012, 69, e4196. [CrossRef] [PubMed]

15. Denvir, M.A.; Tucker, C.S.; Mullins, J.J. Systolic and diastolic ventricular function in zebrafish embryos: Influence of norepenephrine, MS-222 and temperature. BMC Biotechnol. 2008, 8, 21. [CrossRef] [PubMed]

16. Huang, W.-C. Combined use of MS-222 (tricaine) and isoflurane extends anesthesia time and minimizes cardiac rhythm side effects in adult zebrafish. Zebrafish 2010, 7, 297-304. [CrossRef] [PubMed]

17. Romdhani, S.; Vetter, T. Estimating 3D shape and texture using pixel intensity, edges, specular highlights, texture constraints and a prior. In Proceedings of the CVPR 2005 IEEE Computer Society Conference on Computer Vision and Pattern Recognition, San Diego, CA, USA, 20-25 June 2005; pp. 986-993.

18. Pylatiuk, C. Automatic zebrafish heartbeat detection and analysis for zebrafish embryos. Zebrafish 2014, 11, 379-383. [CrossRef] [PubMed] 
19. Müller, I.I. Functional modeling in zebrafish demonstrates that the atrial-fibrillation-associated gene GREM2 regulates cardiac laterality, cardiomyocyte differentiation and atrial rhythm. Dis. Models Mech. 2013, 6, 332-341. [CrossRef] [PubMed]

20. Van Cauwenberge, P. Consensus statement on the treatment of allergic rhinitis. Allergy 2000, 55, 116-134. [CrossRef] [PubMed]

21. Fung, M. Evaluation of the characteristics of safety withdrawal of prescription drugs from worldwide pharmaceutical markets-1960 to 1999. Drug Inf. J. 2001, 35, 293-317. [CrossRef]

22. Olasińska-Wiśniewska, A.; Olasiński, J.; Grajek, S. Cardiovascular safety of antihistamines. Adv. Dermatol. Allergol. Postęp. Dermatol. I Alergol. 2014, 31, 182. [CrossRef] [PubMed]

23. Zhou, Z.; Vorperian, V.R.; Gong, Q.; Zhang, S.; January, C.T. Block of HERG potassium channels by the antihistamine astemizole and its metabolites desmethylastemizole and norastemizole. J. Cardiovasc. Electrophysiol. 1999, 10, 836-843. [CrossRef] [PubMed]

24. Craft, T.M. Torsade de pointes after astemizole overdose. Br. Med. J. 1986, 292, 660. [CrossRef]

25. Yozzo, K.L.; Isales, G.M.; Raftery, T.D.; Volz, D.C. High-content screening assay for identification of chemicals impacting cardiovascular function in zebrafish embryos. Environ. Sci. Technol. 2013, 47, 11302-11310. [CrossRef] [PubMed]

26. Fink, M. A new method for detection and quantification of heartbeat parameters in Drosophila, zebrafish, and embryonic mouse hearts. Biotechniques 2009, 46, 101-103. [CrossRef] [PubMed]

27. Lu, F.; Langenbacher, A.; Chen, J.-N. Transcriptional Regulation of Heart Development in Zebrafish. J. Cardiovasc. Dev. Dis. 2016, 3, 14. [CrossRef] [PubMed]

28. Nerbonne, J.M.; Kass, R.S. Molecular physiology of cardiac repolarization. Physiol. Rev. 2005, 85, 1205-1253. [CrossRef] [PubMed]

29. Milan, D.J.; Jones, I.L.; Ellinor, P.T.; MacRae, C.A. In vivo recording of adult zebrafish electrocardiogram and assessment of drug-induced QT prolongation. Am. J. Physiol. Heart Circ. Physiol. 2006, 291, H269-H273. [CrossRef] [PubMed]

30. Roden, D.M. Drug-Induced Prolongation of the QT Interval. New Engl. J. Med. 2004, 350, 1013-1022. [CrossRef] [PubMed]

31. Devarasetty, M. Optical Tracking and Digital Quantification of Beating Behavior in Bioengineered Human Cardiac Organoids. Biosensors 2017, 7, 24. [CrossRef] [PubMed]

32. Roden, D.M.; Viswanathan, P.C. Genetics of acquired long QT syndrome. J. Clin. Investig. 2005, 115, 2025-2032. [CrossRef] [PubMed]

33. Chiu, P.J. Validation of a [3H] astemizole binding assay in HEK293 cells expressing HERG K+ channels. J. Pharmacol. Sci. 2004, 95, 311-319. [CrossRef] [PubMed]

34. Sallam, K.; Li, Y.; Sager, P.T.; Houser, S.R.; Wu, J.C. Finding the Rhythm of Sudden Cardiac Death. New Oppor. Using Induc. Pluripotent Stem Cell Deriv. Cardiomyocytes 2015, 116, 1989-2004. [CrossRef]

35. Bass, A.S.; Darpo, B.; Valentin, J.P.; Sager, P.; Thomas, K. Moving towards better predictors of drug-induced torsades de pointes. Br. J. Pharmacol. 2008, 154, 1550-1553. [CrossRef] [PubMed]

(C) 2018 by the authors. Licensee MDPI, Basel, Switzerland. This article is an open access article distributed under the terms and conditions of the Creative Commons Attribution (CC BY) license (http:/ / creativecommons.org/licenses/by/4.0/). 